\title{
O papel da informação no processo de capacitação tecnológica das micro e pequenas empresas
}

\section{Paulo César Rezende de Carvalho Alvim}

\section{Resumo}

A necessidade de disponibilizar informações para o setor produtivo tem levado a reflexões e esforços no sentido de atender de forma ampla a esta demanda em uma sociedade do conhecimento.

O artigo foca a relevância da disponibilização de informações no processo de capacitação e inovação tecnológica no segmento das empresas de pequeno porte, especialmente em face da atual relevância econômica e social deste porte de empresa.

Conclui com a indicação de algumas medidas que devem ser perseguidas para ampliar a oferta de informação para as empresas de pequeno porte.

\section{Palavras-chave}

Informação empresarial; Capacitação tecnológica; MPMEs, Informação industrial; Informação tecnológica.

\section{INTRODUÇÃO}

Com o processo de globalização de mercados e a velocidade dos avanços tecnológicos, a busca por informação se tornou alvo comum de toda a sociedade.

Naturalmente, emerge o conceito de uma sociedade da informação, onde ter informação ou ao menos ter garantido o acesso a ela passa a ser um diferencial de uma nova era.

Os esforços que estão sendo feitos no sentido de organizar e analisar dados, de forma a que sejam disponibilizados como informação, com valor agregado, para subsidiar processos de tomada de decisão, é a estrutura orgânica da nova sociedade do conhecimento.

Os avanços ocorridos, nas chamadas tecnologias de informação e na área de comunicação, alavancaram este esforço em uma velocidade de ordem geométrica.

Ao se acessar a Internet, por exemplo, é possível se perder o foco de busca, pela variedade de informações e diversidade de fontes disponíveis.

\section{O NEGÓCIO DA INFORMAÇÃO}

Hoje precisamos passar a ter a preocupação em obter qualidade de informação, e não quantidade.

Este momento de muita informação precisa ser superado rapidamente, para não se perder tempo.

O importante é ter a informação certa, ou melhor dizendo, adequada à determinada necessidade, no tempo correto e a um custo compatível.
Surgem aí novos dilemas, que entendemos se caracterizam como desafios:

\section{Informação adequada a uma necessidade}

O foco de se ouvir a demanda, para se prestarem serviços de informação, exige ajustes nas estruturas formais das unidades e sistemas de informação hoje existentes, que envolvem desde aspectos de decodificação de linguagem até formatação de produtos e serviços de informação.

A máxima de se atender às necessidades do cliente, como pregam os especialistas em qualidade, aplica-se aqui como uma luva.

\section{Informação no tempo correto}

Em uma sociedade em que se antecipar às expectativas do futuro passou a ser um diferencial para o sucesso, o retorno de uma demanda informacional tem de ser na velocidade e nos meios compatíveis com os novos tempos.

Neste caso, é fundamental a incorporação, nas unidades prestadoras de serviços de informação, se possível em tempo real, dos avanços das tecnologias de comunicação e da área de informática.

\section{Custo da informação}

Aqui talvez o maior obstáculo, no caso brasileiro.

Os poucos usuários que sempre tiveram acesso à informação organizada e tratada geralmente por estruturas vinculadas a entidades de âmbito governamental criaram a cultura do acesso e do uso gratuito da informação. 
A informação era um bem, tratado como insumo, não valorado.

A carência de recursos públicos para as atividades de informação, somada ao crescente número de novos usuários e demandantes de produtos e serviços de informação, tem levado as unidades que prestam este serviço a buscar formas de remuneração pelos seus serviços, o que vem sofrendo resistências dos usuários.

A falta de cultura e a inexistência de uma economia da informação têm levado a resistências e ao surgimento de dificuldades da aplicação de regras básicas de oferta-demanda pela aquisição de bens e serviços de valor.

Mas todos estes aspectos precisam ser resolvidos, mesmo surgindo alguns traumas, pois a inserção de uma nação na sociedade da informação leva a que se apliquem práticas antes pregadas no discurso para a educação e a saúde, de universalização e de democratização do acesso. E é neste ponto que fazemos a inserção do nosso tema. Como viabilizar o acesso das pequenas empresas à informação, dentro de uma estratégia de capacitação tecnológica das empresas deste segmento?

\section{INFORMAÇÃO E CAPACITAÇÃO TECNOLOGICA}

Segundo palavras do Papa João Paulo II, "a riqueza das nações funda-se muito mais sobre a propriedade do conhecimento, da técnica e do saber, do que sobre a propriedade dos recursos naturais".

Esta máxima da relevância da tecnologia, em especial como fator alavancador da competitividade e elemento diferenciador que garanta a sobrevivência de empresas e organizações, tem levado a sociedade a desenvolver grandes esforços de capacitação tecnológica.

Entendendo tecnologia como o conjunto de conhecimentos necessários para se conceber, produzir e distribuir produtos e serviços de forma competitiva, o processo de capacitação tecnológica precisa ser compreendido como algo dinâmico e inserido nas estratégias empresariais, que envolvem especificamente a decisão de adaptar ou adquirir tecnologia (via desenvolvimento e/ou compra) para atender à determinada necessidade de uma clientela.

No processo de capacitação tecnológica, é fundamental que se adote uma abordagem sistêmica, baseada na identificação das necessidades do ambiente empresarial de atender às expectativas dos consumidores e clientes de forma rápida e diversificada, como estratégia para manter a competitividade das empresas.

\section{Produtos e serviços competitivos significam empresa competitiva}

A estratégia de uma empresa está diretamente ligada aos objetivos de sua missão e ao seu negócio. A empresa avalia oportunidades e ameaças no seu ambiente de atuação, definindo seus desafios e caminhos a seguir, a partir de seus pontos fortes e fracos. Ao explicitar sua estratégia, a empresa deverá levar em consideração:

- as sinalizações de mercado (considerando os aspectos de produtividade, qualidade, preço, diversidade de produtos e serviços, concorrente, flexibilidade, tempo apropriado e marketing);

- as políticas públicas que afetam o seu dia-a-dia;

- os antecedentes de atuação da empresa nas áreas produtiva e tecnológica;

- a estratégia adotada por outras empresas (benchmarking);

- as externalidades.

Começa a surgir o papel relevante do acesso à informação, pois o acesso às informações anteriormente explicitadas passam a ter papel estratégico na definição dos nortes das empresas.

Quando enfocamos a gestão da atividade produtiva propriamente dita, que envolve as ações de produção e de desenvolvimento tecnológico, tornam-se mais explícitas a relevância e a necessidade de se ter informação.

Focando a ação tecnológica, quando a interface de capacitação se torna mais forte e onde se tem a tarefa de escolher o produto/processo mais adequado, as etapas de projetar, testar e planejar a produção, bem como seus mecanismos de controle, envolvem um nível de especialização e especificidade de busca de informação, que passa a conformar nichos de conhecimento.

Assim sendo, algumas funções se tornam básicas e comuns nas demandas empresariais:

- execução de estudos e sondagens de novas tecnologias (disponíveis ou em desenvolvimento);

- avaliação das tecnologias relevantes à linha de negócio da empresa;

- aquisição de tecnologias a serem utilizadas, entendida de forma ampla (desenvolvimento próprio, ou em parceria e compra de terceiros);

- assimilação dessas tecnologias ao processo produtivo da empresa, incorporando-se como parte de sua competência básica;

- coordenação e gestão da rotina tecnológica da empresa (zelando pelos aspectos de integridade da tecnologia - formação da competência essencial, planejamento do uso futuro desta competência e acompanhamento dos avanços/desdobramentos futuros da tecnologia em questão -, definição clara do uso e emprego da tecnologia e os desempenhos econômico, técnico e comercial).

A formulação da estratégia tecnológica (ofensiva, defensiva, imitativa, dependente, tradicional ou oportunista) incluirá um confronto e análise entre oportunidades/ desafios diante da empresa e de suas reais e atuais possibilidades no campo da tecnologia.

Assim, é necessário ressaltar a importância do estabelecimento de mecanismos de contato com o mundo externo à empresa, sejam eles em nível empresarial, como associações representativas de setores produtivos, ou nos âmbitos nacional e internacional. A existência destes canais de contato é fundamental para o sucesso tecnológico e comercial dos negócios.

E aí surge a relevância das fontes de informação, que, no caso das empresas de pequeno porte, caracterizam-se como um grande gargalo. 
Peter Drucker identifica sete fontes básicas de oportunidades e desafios, que devem ser monitoradas sempre pela empresa:

1) as necessidades de mercado não atendidas;

2) as necessidades dos processos desenvolvidos;

3) as mudanças na estrutura do setor produtivo e/ou do mercado;

4) as mudanças demográficas;

5) as mudanças em percepção, disposição e significado por parte da clientela;

6) os conhecimentos inovadores;

7) o inesperado.

São estas fontes que permitirão atuar na identificação de oportunidades para desenvolver as inovações e caracterizar o diferencial de sucesso empresarial.

O potencial tecnológico da empresa, obtido muitas vezes pela prática de técnicas de auditoria tecnológica, deve ser explicitado por meio de fatores como:

- sensibilização da empresa para a tecnologia;

- sintonia e harmonização entre a es tratégia tecnológica e a da empresa;

- capacitação tecnológica em relação aos concorrentes;

- integração entre P\&D e as demais áreas da empresa;

- adequação da estrutura de PD\&E (pesquisa, desenvolvimento e engenharia);

- adequação das técnicas de gestão de tecnologia.

O potencial tecnológico da organização permitirá aos recursos humanos das empresas conseguir as seguintes respostas, que sintetizam a capacidade instalada e/ou a competência disponível:

1. quais tecnologias são mais promissoras e como incorporá-las aos produtos, processos e serviços da empresa;
2. quais os níveis de competência (conhecimento) necessários;

3. quais são as fontes desta tecnologia (interna e externa) e como obtê-las;

4. quanto investir em tecnologia, em especial em PD\&E;

5. qual o objetivo dentro do processo de inovação(liderar ou seguir);

6. qual a política e a organização para fazer o PD\&E.

Neste ponto, têm-se as respostas básicas de quem e como viabilizar um processo de inovação, capacitação e desenvolvimento tecnológico.

As formas de gestão desta etapa são centradas na utilização de sistemas especializados de informação. $E$ não podem ser de forma diferente, diante das velocidades que se tornam necessárias para as respostas, bem como pela diversidade e multiplicidade de fontes de informação existentes.

Pois é neste momento que as empresas precisam perceber os mecanismos pelos quais a tecnologia é processada, de maneira que ela seja incorporada em sua cadeia produtiva, agregando valor.

A informação, aqui utilizada e analisada, é aquela informação de componente econômico, social e técnico.

A etapa de identificação das tecnologias é uma fase das quais se tem mais estudos a respeito, e Sahal, na Teoria Geral da Evolução dos Artefatos, apresenta cinco princípios como forma de analisar a incorporação de tecnologia nas inovações, de forma global, a saber:

- marcos tecnológicos (desenvolvimentos passados influenciando os novos avanços);

- experiência acumulada (seja por empresas ou pessoas);

- simbiose criativa (duas ou mais tecnologias podendo se fundir de maneira a colaborar no processo de superar os limites ao desenvolvimento de solução de problemas);
- especialização pela escala;

- insularidade tecnológica (obstáculos à transferência de tecnologia entre empresas).

A informação de mercado (informação econômica e social) é a que precisa ser a mais trabalhada, pois ela é relevante para o sucesso das empresas, por ser a responsável pelo êxito de produtos e serviços no mercado.

A estratégia do uso de gestão de sistemas de informação é uma resposta à busca de se minimizar o grau de incerteza e risco no ambiente empresarial. $\mathrm{E}$ muito se tem evoluído nesta área, a partir dos avanços em tecnologia de informação.

Após a fase de identificação (o quê?, por quê?, com quem? e como?), iniciamos o momento da geração da inovação e do desenvolvimento tecnológico propriamente dito, que passa pelas etapas de concepção do novo bem ou serviço e pelos desenvolvimentos que se fazem necessários. É a fase conhecida como PD\&E.

Nesta etapa, tem-se evoluído bastante quanto à oferta de informações, em especial a originada nos fornecedores e provenientes da participação de empresas em missões técnicas e feiras.

Tais etapas têm evoluído bastante, em especial pelo uso intensivo e crescente das ferramentas de tecnologia da informação, assim permitindo otimizar a busca de soluções (usando, por exemplo, técnicas de simulação, CAD etc.) e significando redução de tempo de execução.

\section{O MUNDO DA PEQUENA EMPRESA}

O mundo atual vem assistindo ao desenvolvimento de movimentos e situações em que o ambiente no qual atuam as empresas apresenta-se de forma cada vez mais dinâmico e turbulento, em especial no que tange aos aspectos de mercado, de tecnologias, meio ambiente, transformações políticas, econômicas, culturais e sociais.

Este é o novo contexto de competição das empresas, que tentam, em primeiro lugar, buscar a sobrevivência. 
E sobrevivência das empresas significa cada vez mais aprender a aprender, através de atividades de captação, assimilação e utilização do aprendizado, de forma permanente. Virou uma obsessão!

Precisamos ter uma empresa inteligente, ágil e flexível, atuando como um organismo vivo e pró-ativo.

É neste panorama que surge a oportunidade para a pequena empresa, pois, apesar de ser mais frágil, a empresa de pequeno porte conta com a vantagem de ter a capacidade de reagir mais rapidamente neste novo contexto de mudanças constantes, onde o fazer é sinônimo de aprender.

\section{Caracterização da pequena empresa no Brasil}

No Brasil, a classificação do porte das empresas segue a caracterização pelo número de colaboradores permanentes, com alguns ainda incorporando o fator nível de faturamento.

As empresas de pequeno porte no Brasil são responsáveis por:

- cerca de 4 milhões de empresas constituídas;

- $60 \%$ da oferta total de empregos formais;

- 42\% dos salários pagos;

- 21\% da participação no PIB;

• 96,3\% do número de estabelecimentos.

Diferentemente do papel desenvolvido pelas empresas de pequeno porte no modelo anterior de substituição de importações, no modelo em que se busca competitividade, as pequenas empresas passam a ser elos importantes das cadeias produtivas que buscam a competitividade empresarial brasileira.

Competitividade é o desafio, na década de 90, para as empresas de pequeno porte.

Podemos mesmo afirmar que a competitividade das pequenas empresas brasileiras é essencial ao desenvolvimento do país.
E as empresas de pequeno porte passam a ter presença marcante em diferentes cadeias produtivas, na forma de fornecedores terceirizados e quarteirizados de grandes empreendimentos produtores de bens intermediários e finais, além de atuar como fornecedores de pequenos lotes em nichos de mercado ou em mercados especializados.

A pequena empresa passa a ser vista como um parceiro eficiente e eficaz no processo produtivo, a partir de suas características básicas.

De forma a termos uma visão da especificidade da pequena empresa quanto à capacitação tecnológica, apresentamos, a seguir, um quadro-síntese adaptado de Rothwell.

\begin{tabular}{|ll}
\hline Características & \multicolumn{1}{c}{ Vantagens } \\
\hline $\begin{array}{l}\text { Reação ao } \\
\text { mercado }\end{array}$ & $\begin{array}{l}\text { Capacidade de reagir rapi- } \\
\text { damente às necessidades } \\
\text { de mercado }\end{array}$ \\
Gestão & $\begin{array}{l}\text { Ausência de burocracia } \\
\text { Decisões mais rápidas } \\
\text { Maior propensão ao risco }\end{array}$ \\
& $\begin{array}{l}\text { Sistema de comunicação } \\
\text { informal e eficiente } \\
\text { Solução mais rápida de pro- } \\
\text { blemas internos } \\
\text { Adaptação mais rápida às } \\
\text { mudanças externas }\end{array}$ \\
\hline
\end{tabular}

Recursos

Humanos

Sistema de comunicação externa

Recursos

financeiros

Capacidade de produção

Crescimento

Legislação

Patentes
Legislação

O quadro em foco ressalta que as principais fontes de competitividade das empresas de pequeno porte são a sua capacidade de desenvolver produtos e serviços, sua forma ágil e flexível, bem como a qualidade disponibilizada para a clientela, considerando os aspectos de custo/benefício.

Daí se destacar a relevância da inovação e capacitação tecnológica para a sobrevivência das empresas de pequeno porte.

Além disso, pode-se afirmar que a geração de novas oportunidades de trabalho será extremamente sensível à tecnologia, que reformula os padrões de alocação de recursos de capital e trabalho para a produção de bens e serviços.
Maior desconhecimento das modernas técnicas de gestão

\section{Desvantagens}

Falta de recursos humanos especializados para atender a todas as necessidades internas

Falta de tempo, informações e recursos

Dificuldade de contratar serviços especializados externamente

Dificuldade em conseguir capital Ausência de capital de risco Impossibilidade de reduzir/ratear o risco em um elenco de projetos

Economia de escala pode se tornar uma barreira

Dificuldade de capital para expansão

Lideranças com pouca experiência para lidar com situações mais complexas

Dificuldade em lidar com sistemas de patentes

Não-disponibilidade de tempo e recursos para processos litigiosos

Dificuldade em lidar com legislações mais complexas 


\section{A PEQUENA EMPRESA E A TECNOLOGIA}

A vocação natural da pequena empresa é aplicar tecnologias existentes de forma original em novos produtos e serviços.

Isto decorre de a atitude da empresa de pequeno porte ter baixa atuação em $P D \& E$, que, neste caso, é fruto direto de problemas de escala e de sua capacidade de investimento.

Recente pesquisa feita pela Confederação Nacional da Indústria, a pedido do Ministério da Ciência e Tecnologia, concluiu que a maioria das micro e pequenas empresas brasileiras está na contramão da economia mundial no que diz respeito à inovação tecnológica.

Só $6 \%$ das micro e $14 \%$ das pequenas empresas investem mais de $5 \%$ do seu faturamento em inovação, atividade vital para a manutenção da competitividade.

No caso brasileiro, está aumentando o fosso tecnológico entre as empresas de pequeno porte e as grandes, criando dificuldades futuras, inclusive no que tange à incapacidade de atendimento das demandas de grandes empresas como fornecedores.

Em outra pesquisa feita pela Coppe/ UFRJ junto a pequenas empresas de incubadoras de base tecnológica, identificaram-se as seguintes dificuldades:

- fraca demanda das empresas em serviços tecnológicos;

- dificuldade de encontrar parceiros no processo de produção;

- dificuldade de conseguir fornecedores confiáveis;

- dificuldade de financiamento da produção (capital de giro);

- acesso limitado a financiamento público para iniciar produção;

- inexperiência em termos de comercialização;

- identificação limitada de potenciais usuários e compradores;
• invisibilidade da empresa no mercado; • clientes;

- mercado de difícil identificação;

- fornecedores.

- falta de experiência gerencial.

Entretanto, estudos feitos no âmbito da América Latina ressaltam que a capacidade de se anteciparem as necessidades do ambiente onde está inserida é talvez a principal vantagem da empresa de pequeno porte neste cenário sombrio. Isso, porque, ao identificar oportunidades, as empresas concentram seus recursos e alcançam com rapidez o mercado, utilizando-se das suas características de velocidade, dedicação e espírito empreendedor.

A seguir, são apresentados resultados de uma pesquisa realizada na Venezuela, onde foram identificados aspectos relevantes do porquê de as empresas de pequeno porte inovarem e investirem em tecnologia. Estes resultados, com certeza, podem ser estendidos à realidade brasileira.

1) Fatores mais importantes que levam as pequenas empresas a se motivarem para a tecnologia:

- ter liderança de mercado;

- melhorar a qualidade de seus produtos/serviços;

- criatividade dos empresários;

- explorar nichos de mercado;

- exportar;

- trabalhar em equipe.

2) Fontes internas à empresa de conhecimento:

- seus recursos humanos;

• serviços de informação;

- experiência da empresa e de seus recursos humanos;

- P\&D.

3) Fontes externas à empresa:

- mercado;
4) Influência do entorno competitivo no processo de capacitação tecnológica:

- oportunidade de mercado;

- possibilidade de alianças estratégicas.

5) Obstáculos ao esforço de capacitação tecnológica:

a) Internos à empresa:

- carência nas técnicas de mercado;

- pouca capacidade financeira;

- falta de estudos de mercado;

- falta de liderança;

- capacidade produtiva limitada e, às vezes, de difícil desmobilização/modificação.

b) Externos à empresa:

- sofisticação da demanda;

- falta de informação de mercado;

- pouca aceitação do produto no mercado;

- necessidade de alta competência;

- preço do produto;

- falta de informação tecnológica.

6) Estratégias empresariais adotadas pelas empresas de pequeno porte:

a) Estratégia competitiva:

- desenvolvimento de novos produtos e mercados;

- desenvolvimento de nichos de mercado;

- competência em custos;

- integração horizontal;

- agressividade, independência e risco. 
b) Estratégia tecnológica:

- adaptação de tecnologias;

- compra de tecnologia.

Em outra pesquisa de 1996, a cargo da CNI e Ciet/Senai, no âmbito do PACTI-MCT, foi identificado que as principais fontes de informação de interesse empresarial são fornecedores, feiras e publicações técnicas.

Entretanto, o quadro não é tão pessimista, pois, segundo levantamentos do Sebrae, tem sido crescente a demanda por informações tecnológicas pelos pequenos empresários. A demanda por informação é o primeiro passo para a inserção, na empresa, da preocupação com o aspecto de capacitação tecnológica.

Inclusive, hoje, já se tem a situação da empresa de pequeno porte com noção da importância do processo de desenvolvimento tecnológico. Há bem pouco tempo, a pequena empresa brasileira tratava tecnologia como algo sofisticado e distante de sua realidade e necessidade, relacionando-a sempre com a empresa de maior porte.

Hoje, a maioria das empresas que procura os Sebraes nos estados ainda busca ajuda para modernização, o que envolve ações de difusão tecnológica e de atendimento de demandas de inovações simples (incrementais).

O papel do Sebrae tem sido o de mostrar o caminho das pedras.

$E$, em um processo de parceria com entidades tecnológicas (universidades, centros e institutos de pesquisa e desenvolvimento, escolas técnicas, prestadores de serviços tecnológicos e outros) e com especialistas, o Sistema Sebrae tem executado relevantes serviços em prol da alavancagem tecnológica da empresa de pequeno porte.

Pesquisa feita com base nos relatórios das atividades de apoio tecnológico prestado ou viabilizado pelo Sebrae às pequenas empresas identificou números significativos de atendimentos com resultados de melhoria de processo, produto e métodos de gestão, além de crescente presença de projetos de inovação, principalmente de novos produtos.
Deve ser destacado que a intervenção de apoio do Sebrae se faz sempre com o uso de terceiros, o que, de alguma forma, é uma estratégia de agregação de conhecimento à empresa de forma otimizada, pois se utiliza da capacidade de infra-estrutura de C\&T instalada no país, reduzindo-se os custos fixos de manutenção de equipes especializadas nas empresas de pequeno porte.

\section{A INFORMAÇÃO E A PEQUENA EMPRESA}

Quando se fala de informação, inicia-se um processo em que é difícil colocar limites.

Vamos nos ater, neste trabalho, no espectro de informações de interesse empresarial, envolvendo informações do ambiente dos negócios e suas oportunidades e as intrínsecas à empresa e ao setor a que pertence, passando por processos de rotina (administrativa, financeira, gerencial, tecnológica, comercial etc.), planejamento, estratégia e desafios.

Com o avanço das tecnologias de informação, muito tem sido feito no sentido de disponibilizar informações para o setor produtivo, especialmente em meio eletrônico.

Tem crescido o número de serviços de informação e de bases de dados à disposição dos empresários.

Peter Drucker em 94, quando esteve no Brasil, disse nos que o executivo, para sobreviver e prosperar no ambiente das empresas baseadas em informação, precisa aprender a aceitar a responsabilidade pela informação, dar à informação o formato de uma ferramenta de compreensão, contrabalançar as informações externas com as internas e definir precisamente o que são informações.

Em estudo de 97, o IBICT/CNPq fez um extensivo levantamento de fontes de informação, entendida como toda ou qualquer forma de conhecimento relevante para operar um empreendimento (conhecimento técnico, econômico, social, cultural, mercadológico, legal etc.).
O provimento destas informações, em bases sistemáticas, ao setor produtivo do país tem se constituído em uma das áreas prioritárias de atuação das entidades de informação.

A implantação de serviços de informação para o setor produtivo em forma de redes se intensificou a partir da criação da Rede de Núcleos de Informação Tecnológica do Subprograma de Tecnologia Industrial Básica do PADCT nos anos 80 .

Cabe destacar que, no I PBDCT, em 72, foi instituído o Subsistema de Informação Tecnológica e Industrial, transformado, no II PBDCT, em Rede de Informação Tecnológica Industrial em 76. Ou seja, o arcabouço institucional foi concebido de forma correta há bastante tempo.

Entretanto, o momento não era o adequado, em função do modelo de desenvolvimento econômico praticado (substituição de importações).

Os anos 80 são os anos de reestruturação do processo econômico e produtivo, com a incorporação de visões de mercados globais e de competitividade.

Neste ambiente, informação passa a ser fator diferencial de sucesso.

Daí se expandirem sistemas onde se destacam os do Sebrae, CNI, Senai, Dampi, IEL, universidades, Embrapa, associações de classe, institutos de pesquisa em C\&T (liderados pela Abipti) e suas interfaces com sistemas estrangeiros (TIPs, Latin, Intib/Unido, Unesco, Olamp e outros) e oficiais de informação (Simpri, Inpi, Inmetro e outros). Um esforço recente que deve ser destacado é o dos trade points, instrumentos integradores da oferta de oportunidades (via acesso à informação estratégica), que atuam somando.

Cabe destacar que, no caso do Sistema Sebrae, seu êxito tem base principalmente no aspecto de capilaridade de atendimento, em especial o serviço de informação prestado pela sua Rede de Balcões, totalizando cerca de 800, em todo o território nacional. 
Democratizar o acesso à informação torna-se uma das prioridades nacionais.

É a democratização das oportunidades.

Os serviços de informação no país, hoje, são realizados por instituições públicas (em sua maioria) e privadas, atuantes em todos os estados brasileiros, com grande concentração no Sul-Sudeste do país.

Muitas destas instituições atuam como centros de referência, interligando inúmeras instituições em uma ampla rede de alcance mundial.

São redes de redes. Temos uma teia.

As tecnologias de informação, em especial a Internet, servem como grande alavancador deste complexo sistema.

Os serviços e produtos de informação oferecidos por estas instituições podem ser classificados como básicos e especializados (os de maior valor agregado).

Nesta década, estamos presenciando a especialização dos serviços de informação, seja pela abordagem de foco setorial (especialização da clientela), seja pela disponibilização de produtos e serviços de maior valor agregado (especialização pela oferta).

Neste sentido, verificamos um movimento de especialização de redes de informação de interesse empresarial, envolvendo os sistemas Senai, IEL, Rede de Núcleos de Informação Tecnológica, Rede Antares, Rede de NITs do Competir, SIE dos Sebraes do Norte e Nordeste do país, Infovia CNI, entre outras.

\section{INFORMAÇÃO E A CAPACITAÇÃO EMPRESARIAL NO MUNDO DOS PEQUENOS NEGÓCIOS}

Na escala de agregação do conhecimento a bens e serviços, o primeiro estágio é o de identificar, coletar, organizar e tratar dados, transformando-os em informação.

Muito se tem evoluído neste âmbito, em especial dentro das esferas internas dos serviços de informação.
Poderíamos dizer que se atua no foco da oferta da informação.

O grande desafio é disponibilizar a informação para o usuário de forma adequada, para que venha subsidiar um processo de tomada de decisão que se transforme em benefício para a sociedade.

Transformar informação em conhecimento que se converta em bem econômico e social é o desafio.

E este desafio só pode ser superado pela integração oferta/demanda.

Potencializar oportunidades, a partir da utilização de capacidades disponíveis e acessíveis, torna-se o grande negócio de quem atua em prestação de serviços de informação.

Alavancar negócios, por meio do acesso adequado e antecipado às oportunidades, é a forma de colaborar para a sobrevivência e o desenvolvimento empresarial.

Os trabalhos desenvolvidos em prospecção e em gestão estratégica do conhecimento precisam ser ampliados e mais disseminados, inclusive no que tange à formação de massa crítica.

\section{POR QUE A PEQUENA EMPRESA TEM DE SE CAPACITAR TECNOLOGICAMENTE}

A título de ilustração, apresentaremos a listagem dos problemas mais comuns da empresa de pequeno porte que a levam a buscar ação de capacitação e inovação tecnológica:

- dificuldade de acesso à tecnologia;

- prática de técnicas de produção rudimentares;

- uso de mão-de-obra pouco qualificada;

- falta de aplicação de métodos de trabalho;

- subaproveitamento de seus equipamentos-ociosidade;

- prática de processo produtivo pela forma empírica;
- desperdício de matéria-prima;

- elevados índices de retrabalho;

- aumento do custo de fabricação;

- clientela insatisfeita;

- crescimento estagnado;

- falta de visão com relação às possibilidades de investimento para a melhoria de produtividade.

Vê-se que a problemática das empresas de pequeno porte está sendo colocada timidamente no campo dos estudos de gestão da inovação e de estratégia tecnológica.

A ênfase dada, tradicionalmente, a algumas características das empresas de pequeno porte rotulou-as como receptoras passivas e episódicas de tecnologia.

Emerge, a partir da década de 80, um interesse no sentido de incorporar as empresas de pequeno porte como atores dinâmicos no processo de inovação tecnológica.

Tal fato se deve ao esgotamento do modelo de produção fordista, segundo o qual o crescimento econômico estava baseado em grandes empresas, intensivas em capital, com significativas economias de escala, forte capacidade interna de P\&D e situação predominante de oligopólios de mercado.

Assim, o papel econômico das empresas de pequeno porte passou a ser reavaliado em função de suas características básicas, já exploradas neste trabalho.

Quando o que vale é o human capital, a empresa de pequeno porte floresce.

Hoje, as empresas de pequeno porte são vistas como agentes de difusão das novas tecnologias e das inovações, sendo que elas têm necessidade de ser apoiadas por medidas adequadas, para fortalecer e ampliar a sua capacitação tecnológica e seu potencial inovador.

Timidamente, nas últimas duas décadas, surgiram instrumentos de apoio à capacitação tecnológica das empresas de pequeno porte no Brasil. 
O que se percebe é a existência de demanda concreta e crescente para instrumentos de apoio à capacitação tecnológica da pequena empresa

No que tange ao apoio à inovação e capacitação tecnológica, os instrumentos hoje operados pelo Sebrae, Finep e Fundações Estaduais de Apoio à Pesquisa apresentam resultados ainda insatisfatórios, apesar de exitosos.

\section{UM CAMINHO A PERSEGUIR E CONQUISTAR}

Entendemos que o apoio, como forma de alavancar e dar start ao processo de capacitação e inovação tecnológica na empresa de pequeno porte, deve ser desenvolvido prioritariamente e em linhas gerais, com as seguintes ações:

\section{Difusão de informações de interesse empresarial}

Facilitar o acesso da pequena empresa às informações de oportunidades de negócio, fornecedores (matérias-primas, equipamentos, tecnologia, serviços tecnológicos etc), fontes de tecnologia, mercado, comércio internacional, especificações de mercado comprador, legislação etc.

Neste aspecto, é fundamental o processo de democratização do acesso à informação, incluindo os pontos referentes à adequação de linguagem ao público-alvo e aos modos de comunicação.

\section{Information role in the process of technological training of micro and small size interprise}

\section{Abstract \\ The need to make information available to the production sector has led to reflections aiming at meeting those needs broadly in a Knowledge Society. \\ This article focuses on the relevance of the availability of information process of technological qualification and innovation for small businesses, cation and mainly in view of current those businesses's economic and social significance. \\ It includes by suggestion a few measures that shoulder pursued to expand the offer of information for small businesses.}

\section{Keywords}

Companies information; Technological capacity; Small businesses; Industrial information and technological information.
Devem ser disponibilizados mecanismos de baixo custo que atendam às necessidades de informação deste porte de empresa, preferencialmente de foco setorial.

Para tanto, é fundamental ampliar as redes que hoje prestam estes serviços, em especial dentro do foco especializado, corrigindo e buscando-se melhor equilíbrio na sua distribuição espacial.

Outro aspecto que deve ser trabalhado é o da publicidade destes serviços, pois, apesar da unanimidade de sua relevância, continuam desconhecidos para grande parcelas de suas clientelas.

\section{Apoio a estudos de viabilidade de disponibilizar produtos/serviços diante das oportunidades sinalizadas pelo mercado}

Financiar estudos de mercado, econômicos, de prospecção tecnológica e mercadológia, bem como a participação em feiras e eventos (missões técnicas).

Viabilizar o acesso a sistemas de informação estratégica, nas áreas de tecnologia e de mercado.

Desta forma, estaremos dando tratamento igualitário às empresas de pequeno porte ao tratar as oportunidades, como oportunidades.

Ações de pré-investimento, nos moldes preconizados nos apoios do Projeto Alfa e do programa da Fapesp, devem ser incrementados.

As empresas de pequeno porte que têm como principais características o papel de geração de novos postos de trabalho, de difundir, de forma mais democrática, os avanços tecnológicos e de contribuir efetivamente para o bemestar social e econômico, estimulando o empreendedorismo, precisam de apoio de conteúdo, e não de ação paternalista.

Assim, deve ser estimulado o papel de inovar e criar da pequena empresa, em qualquer tipologia de inovação e capacitação tecnológica, via o processo de democratizar o acesso à informação de interesse empresarial.

\section{BIBLIOGRAFIA}

1. ALVIM, P. Inovação na pequena empresa: como alavancar. São Paulo, PROTAP 97, 1997

2. CNI - CIET - SENAI Demanda por informação tecnológica pelo setor produtivo: pesquisa. Rio de Janeiro, 1996

3. CNI - MCT Estudo da demanda do setor privado por investimentos em tecnologia: pesquisa 1997 - 2001. Rio de Janeiro, 1997

4. CNPq - IBICT A informação tecnológica no Brasil: relatório preliminar. Brasília, 1997.

5. DRUCKER, P. The new managemente and the new company. São Paulo, HSM, 1994.

6. DRUCKER, P. The practice of management [s.l.: s.n.] 1954

7. FUNDAÇÃO DOM CABRAL Internacionalização de empresas brasileiras. Belo Horizonte, 1996

8. IBARRA, R.C., REBOLLEDO, J.L.S., MARTINEZ, R.L. Estabelecimento de lineamientos generales de política para la creacion de capacidades tecnológicas internas em pequenas y medianas empresas mexicanas. São Paulo, [s.n.], 1996

9. KRUBLIANSKAS, I Formando a pequena e média empresa competitiva: como inovar e sobreviver em mercados globalizados. São Paulo, [s.n.], 1996.

10. KRUGLIANSKAS, I, SBRAGIA, R. Função tecnológica em pequenas empresas: estudo de algumas dimensões críticas para sua implantação. São Paulo, [s.n.], 1995.

11. MACULAN, A.M. Estratégia tecnológica de micro-empresas localizadas numa incubadora. São Paulo, [s.n.], 1996.

12. MACULAN, A.M. Estratégias de inovação tecnológica das pequenas e médias empresas. Salvador, [s.n.], 1993.

13. ROTHWELL, R. Small Jirms innovation and industrial change. [s.I.:s.n.], 1989.

14. SAHAL, D. Paherns of technological innovation. [s.I.: s.n.], 1981.

15. SALAZAR, D.V., ARRIETA, M., SALAZAR O. Iniciativas inovadoras en la pequeña empresa - estúdio de casos. São Paulo, [s.n.], 1996.

Artigo aceito para publicação em 10-12-97

\section{Paulo César Rezende de Carvalho Alvim}

Técnico de Projetos e Programas da Unidade de Negócios Internacionais da Finep.

E-mail:palvim@gabi.finep.gov.br 